\title{
Current information about vaccination practice in pediatric rheumatic diseases and recommendations for future applications
}

\author{
Ceyhun Açarı, Erbil Ünsal \\ Division of Rheumatology, Department of Pediatrics, Dokuz Eylül University Faculty of Medicine, Izmir, Turkey. \\ E-mail: erbil.unsal@deu.edu.tr \\ Received: 12th August 2017, Accepted: 17th September 2017
}

SUMMARY: Açarı C, Ünsal E. Current information about vaccination practice in pediatric rheumatic diseases and recommendations for future applications. Turk J Pediatr 2017; 59: 357-368.

Pediatric patients with autoinflammatory or rheumatic diseases are at increased risk of infections; therefore, safe and effective immunizations are crucial in the management of these group of patients. Current aggressive treatments involving the early use of immunosuppressive drugs and biological agents have further increased the susceptibility to infections in this group of patients. Therefore, effective and safe vaccination with adequate serological responses is important. In patients with rheumatic diseases, immunogenicity of a vaccine can differ from the healthy population, because of the disease itself or the immunosuppressive treatment received. Moreover, possible effects of vaccination on the underlying disease should be considered. In general, live attenuated vaccines should not be administered when highdose immunosuppressive drugs are used for immunosuppressed patients. Inactivated vaccine agents have proven to be generally safe in patients with RD. The immune-modulating of biologic agents effects can last for weeks to months after discontinuation, depending on their half lifes. Also, live virus vaccines are contraindicated during therapy and for weeks to months following discontinuation of the biologics. The aim of this review is to cover the current information about vaccination practice in pediatric rheumatologic diseases and to give recommendations for future applications.

Key words: pediatric rheumatic disease, vaccination, live-atenuated vaccine, biologicals.

Vaccination is one of the most important successes in the medicine history. Many vaccines are included in national immunization programs world-wide. ${ }^{1}$ World Health Organization's (WHO) guidelines about vaccination and/ or national vaccination guidelines should be followed for the vaccination. The primary vaccination schedule is applied especially during childhood and adolescence when the highest prevalence of certain rheumatic diseases occur. ${ }^{2}$

Pediatric patients with autoinflammatory or rheumatic diseases are at increased risk of infections; therefore, safe and effective immunizations are crucial in the management of these group of patients. ${ }^{3}$ Current aggressive treatments involving the early use of immunosuppressive drugs and biological agents have further increased the susceptibility to infections in this group of patients. ${ }^{4-6}$ Therefore, effective and safe vaccination with adequate serological responses is important.

The aim of this review is to cover the current information about vaccination practice in pediatric rheumatologic diseases and to give recommendations for future applications.

\section{Vaccination in Pediatric Rheumatic Diseases}

The immunogenicity concept means the immune response induced by vaccination and is often used to assess the efficacy of vaccination in pediatric patients with rheumatic diseases (RD). The response is evaluated by using vaccine-specific geometric mean antibody titers (GMT) or concentrations (GMC), seroconversion rates and/or seroprotection rates. The immunogenicity of the vaccine 
depends on protection and immunological response of each pathogen due to the interaction between the humoral and cellular immune system. ${ }^{7-9}$ In patients with rheumatic diseases, immunogenicity of a vaccine can differ from the healthy population, because of the disease itself or the immunosuppressive treatment received. ${ }^{10}$ Moreover, possible effects of vaccination on the underlying disease should be considered. In general, live attenuated vaccines should not be administered when high-dose immunosuppressive drugs are used for immunosuppressed patients (solid organ transplantation, hematologic malignancy, immunodeficient patients). ${ }^{11,12}$

Inactivated vaccine agents have proven to be generally safe in patients with $\mathrm{RD}$, but some studies in adults have shown that disease activation might be triggered after vaccination. ${ }^{13}$

Immunoglobulin administration may reduce the effectiveness of live vaccines. Therefore, it is necessary to apply live vaccines (i.e. measles, varicella vaccine) at least 3 months following the immunoglobulin administration. If immununglobulin administration is required within 14 days of live vaccine administration, these vaccinations need to be repeated later.

Receiving daily corticosteroid treatment at a dose of $20 \mathrm{mg}$ (or $>2 \mathrm{mg} / \mathrm{kg} /$ day for patients $<10 \mathrm{~kg}$ ) of prednisone or equivalent for $>14$ days or receiving certain biologic drugs (i.e. adalimumab, certolizumab, infliximab, etanercept, golimumab, rituximab) are regarded as "high-level immunosuppression" by the Infectious Diseases Society of America guidelines. "Low-level immunosuppression" is accepted as receiving prednisone at a dose of $0.5 \mathrm{mg} / \mathrm{kg} /$ day, methotrexate (MTX) at doses of less than $0.4 \mathrm{mg} / \mathrm{kg} /$ week or azathioprine at less than $3 \mathrm{mg} / \mathrm{kg} /$ day. Immunization should be initiated prior to immunosuppression. Live vaccines should be administered $\geq 4$ weeks prior to initiation of immunosuppression and should not be given within 2 weeks before initiation. Inactivated vaccines should be administered $\geq 2$ weeks prior to immunosuppression. ${ }^{11,14}$

Biologics, also known as cytokine inhibitors, are drugs used to treat immune mediated conditions, including juvenile idiopathic arthritis, inflammatory bowel disease and autoinflammatory diseases. Their immunemodulating effects can last for weeks to months after discontinuation, depending on their half lifes. Patients treated with biologics are at increased risk of infections caused by mycobacteria, molds, fungi, legionella and other intracellular pathogens. Also, live virus vaccines are contraindicated during therapy and for weeks to months following discontinuation of the biologics. Inactivated vaccines are recommended during therapy as recommended for annual immunization schedule and should not be withold because of a concern of an exaggerated inflammatory response. ${ }^{11}$

\section{Inactive Vaccines}

It is recommended to follow the national vaccination guidelines for vaccination with inactive vaccines such as vaccines against cholera, diphtheria, Haemophilus influenzae type B (Hib), hepatitis A virus (HAV), hepatitis B virus (HBV), Japanese encephalitis, pertussis, pneumococci, poliovirus and meningococci, rabies, tetanus, tickborne encephalitis and typhoid fever, in pediatric patients with RD. ${ }^{12}$

\section{Influenza vaccine}

There are two different influenza vaccines as inactive and live-atenued vaccine. Seasonal influenza virus vaccination is recommended for children between 6 months and 18 years of age who are at high risk for influenza complications. The annual influenza vaccine should be considered in all patients. General recommendations indicate that "immunosuppressed" children should receive annual "inactive" influenza vaccines. ${ }^{15}$

The incidence of influenza infection is not yet known for the pediatric patients with RD. ${ }^{10}$ There are studies indicating the risk of influenza in elderly patients with RD, and a beneficial effect of vaccination on admission for pneumonia/flu or death. ${ }^{16}$ Six studies involving 353 patients are in favor of the safety and immunogenicity of vaccination against seasonal influenza in pediatric patients with RD. ${ }^{17-23}$ The studies including steroids (from $\leq 0.5 \mathrm{mg}$ / $\mathrm{kg} /$ day $^{18-20}$ to $>2 \mathrm{mg} / \mathrm{kg} / \mathrm{day}^{22}$ ), and disease modifying antirheumatic drugs (DMARDS) such as MTX $^{18,19}$, azathioprine ${ }^{18,19,22}$, cyclophosphamide ${ }^{19}$, cyclosporine ${ }^{18,19}$, 6-mercaptopurine ${ }^{22}$, mycophenolate mofetil ${ }^{19}$, tacrolimus $^{19}$ and chlorambucil ${ }^{21}$ showed good immunogenicity following influenza vaccine.

Shale et al. ${ }^{24}$ have evaluated the effect of annual 
inactivated influenza immunization under antiTNF therapy (i.e. etanercept, adalimumab or infliximab) in adult and pediatric patients and reported that it is safe and effective. However, Kapetanovic et al. ${ }^{25}$ demonstrated lower number of responders to the vaccine in RA patients receiving TNF blockers alone or in combination with MTX and/or other DMARDS.

Seasonal influenza vaccine is safe and immunogenic in adult and pediatric patients with RD and it is recommended annually.

\section{Hepatitis B vaccine}

Highly effective and safe hepatitis A (HepB) vaccines produced by recombinant DNA technology are licensed in the United States in single-antigen formulations andas components of combination vaccines. Using immunosuppressant drugs or biologic agents rise the risk of $\mathrm{HBV}$ reactivation which causes liver failure and death in patients with cancer and RD. ${ }^{26,27}$ Hence, patients with RD should be investigated for current or past HBV infection by $\mathrm{HBs}$ antigen, anti-HBs antibodies, and anti-HBc antibodies before starting immunosuppressants or biologic agents. ${ }^{28}$

In most studies, vaccination against hepatitis B virus was found to be safe and immunogenic. ${ }^{28-31}$ Studies on HBV vaccines in pediatric patients with RD showed that most of the patients had adequate antibody levels following vaccination. ${ }^{29,32-35}$ Humoral response after HBV vaccination should be checked, because antibody titers may fall below their protective levels over time, and a booster dose is needed. ${ }^{10,32,33}$ The literature is controversial on the safety of HBV vaccine in autoimmune diseases. Several autoimmune manifestations have been described after HBV vaccine, such as vasculitis, SLE, RA, seronegative arthropathies. ${ }^{6}$ A study reported recurrence or worsening of RD following HBV vaccination, including four cases of SLE. ${ }^{36}$ Another case control study with 260 SLE patients failed to describe the HBV vaccine as a risk factor for the development of this disease. ${ }^{37}$ On the other hand, a study with recombinant $\mathrm{HBV}$ vaccine showed protective responses in 38 out of 39 JIA patients and no increase in disease activity was observed. ${ }^{29}$

\section{Hepatitis A vaccine}

The vaccines are produced from antigenic component of hepatitis $A$ (HepA) virus, which is propagated in human fibroblasts purified from cell lysates, formalin inactivated, and adsorbed to an aluminum hydroxide adjuvant. HepA vaccines are licensed for people 12 months and older and have pediatric and adult formulations that are administered in a dose schedule. Russo et al. ${ }^{38}$ reported two JIA cases who developed macrophage activation syndrome following hepatitis A infection, and stated that one of the patients died. Erguven et al. ${ }^{39}$ stated hepatitis $A$ vaccine was safe in patients with JIA. The response to vaccine did not differ between healthy children and patients with JIA, except in children with active systemic JIA receiving anti-TNF $\alpha$ drugs.

\section{Pneumococcal vaccines}

Pneumococcal vaccines are two types as 13-valent pneumococcal conjugate vaccine (PCV13) and 23-valent pneumococcal polysaccharide vaccine (PPV23). PCV13 is recommended four times in early childhood. ${ }^{40}$ High-risk patients between 2-5 years need at least one PCV13 vaccine depending on the previous number of pneumococcal conjugate vaccines received. ${ }^{41}$

PCV is administered up to two years in children, and up to five years in immunosuppresent patients. ${ }^{42}$ High-risk patients between 6-18 years, need PCV13, if they have not received it before in early childhood. ${ }^{43}$ Most studies supported the safety and immunogenicity of PPV and PCV vaccines in pediatric patients with RD. ${ }^{12,30,44}$ The responses to 7 -valent pneumococcal conjugate vaccine was investigated in one study. Significantly lower antibody concentrations to three serotypes were found in patients on anti-TNF $\alpha$ treatment. However, response and protection rates were found similar. PCV7 vaccination was deemed safe and immunogenic in JIA patients, although anti-TNF $\alpha$ treatment may reduce antibody concentrations. ${ }^{17,44}$

The Centers for Disease Control and Prevention (CDC) recommends PCV13 followed by the PPV23 to high-risk children. ${ }^{41}$

\section{Meningococcus vaccines}

Diverse types of vaccines against Neisseria meningitidis exist such as polysaccharide vaccines against serogroups $\mathrm{A}, \mathrm{C}, \mathrm{Y}$ and $\mathrm{W}-135$, and the monovalent or tetravalent conjugate vaccines. ${ }^{17}$

The Meningococcus $C$ conjugate vaccine neither exacerbates JIA disease activity, nor increase 
relapse frequency with sufficient antibody levels, even in patients receiving aggressive immunosuppressive treatment. Hence, patients with JIA can be vaccinated safely and effectively with the MenC conjugate vaccine. Huijssoon et al. ${ }^{45}$ evaluated antibody titers in 234 patients with JIA following vaccination against Meningococcus, all of which seem to be adequately protected against meningococcal serogroup C disease, irrespective of the immunosuppressive treatment given.

According to Stoof et al. ${ }^{46}$ adolescent patients with JIA showed the highest MenC-specific IgG antibody responses upon MenC vaccination. Persistence of MenC-specific IgG antibodies in JIA patients is similar to healthy controls, but treatment with biologics may induce accelerated antibody waning, resulting in unprotected patients who may need revaccination.

Vaccination against meningococcal disease in JIA patients is also recommended in the European League Against Rheumatism (EULAR) guidelines. ${ }^{12}$

\section{Diphteria-Pertussis-Tetanus vaccine}

In many countries tetanus-diphtheria vaccination combined with pertussis vaccine has been added to the national vaccination program. Several combinations such as tetanus- diphtheriaacellular pertussis (TDaP), tetanus-diphtheriawhole cell pertussis (TDwP), tetanus toxoid (TT) and tetanus-diphtheria (TD) exist. Because of the adverse events of whole cell pertussis vaccinations, acellular pertussis vaccines are preferred in many countries. ${ }^{17}$

The tetanus and diphtheria toxoids, and antipertussis compounds vaccines are safe for children and adults with RD. Patients should be vaccinated according to the vaccination schedule used for healthy children. ${ }^{47,48}$ The classical vaccine (DPT) has cellular components that can trigger the development of adverse reactions; however, it is recommended in patients with RD, even those on immunosuppressive therapy. ${ }^{49,50}$ Four studies including 95 patients and 125 healthy controls show the immunogenicity of the TT or TD vaccines. High antibody concentrations against tetanus (3 studies) and diphtheria (1 study) were found. ${ }^{21,51-53}$ In these studies, similar antibody levels were detected compared to the healthy control group, even in patients using DMARDS.
Miyamoto et al. ${ }^{51}$ reported lower antibody concentrations and seroprotection rates in 18 SLE patients who received tetanus vaccine. The time elapsed since the last vaccination was found similar between patients and controls in this study. In another study, Heijstek et al. ${ }^{54}$ found lower protective antibody levels against diphtheria and tetanus in 400 patients with JIA compared to healthy controls. According to this study, while the vaccination responses were initially similar, the persistence of specific antibody concentrations in pediatric patients with $\mathrm{RD}$ reduced with time. The influence of biologics on antibody responses to TT have been studied only in adults, and it is found that rituximab had no influence on antibody responses to TT in patients with RA receiving weekly MTX. ${ }^{55}$

Healthy volunteers who were administered a single dose of abatacept before TT vaccination showed significant antibody responses, but lower antibody titers than those who have not received abatacept. 56

DT/TT is usually effective and safe in patients with RD using corticosteroids, immunosuppressants, and biologics, but some reports demonstrated lower antibody responses in these conditions. Hence, antitetanus immunoglobulin may be considered in pediatric patients with RD receiving highdose corticosteroid, immunosuppressants, or biologics drugs. ${ }^{28}$

Koscheva et al. 57 investigated 55 pediatric patients with RD who were in clinical and laboratory remission, however, some of them were still receiving NSAIDs or immunosuppressive maintenance therapy. According to this study, diphtheria vaccination was immunogenic and safe in $95 \%$ of the children. Protective antibody levels were maintained for a long time, and simultaneous use of immuno-suppressive treatment did not inhibit the production of protective antibodies.

\section{Human papilloma virus vaccine}

There are two types of human papilloma virus (HPV) vaccines: the quadrivalent (qHPV) vaccine (against HPV 6, 11, 16 and 18) and the bivalent (bHPV) vaccine (against HPV 16 and 18). ${ }^{10}$ In a study including 63 JIA patients and 48 healthy controls, all participants were found seropositive up to 12 months 
following bHPV vaccination. Antibody levels were found to be lower in patients than controls, but no statistical significant difference was determined in results over time. ${ }^{58}$ Sufficient immunogenicity of HPV vaccination has a specific importance in patients with SLE, as these patients have a high risk of persistent HPV infections. ${ }^{59}$ Two studies showed that the majority of jSLE patients who were vaccinated against HPV was seroconverted. ${ }^{58,60}$ However, lower antibody concentrations were found in six jSLE patients compared to the controls. Lower GMCs were detected for antibody titers against HPV in patients than in healthy controls. ${ }^{61}$ Based on these results, long-term protection against HPV infections in SLE patients is not clear. Future controlled studies in larger group of jSLE patients are needed to evaluated the immunogenicity of the HPV vaccine in this group. ${ }^{10}$

\section{Live-Attenuated Vaccines \\ Bacillus Calmette-Guérin vaccine}

Glucocorticosteroids, DMARDS (especially MTX and leflunomide) and/or anti-TNF $\alpha$ agents (infliximab and adalimumab) increase the risk of tuberculosis in adult rheumatic patients. ${ }^{17}$ This data is not valid for pediatric population and there are only a few cases of tuberculosis associated with anti-TNF $\alpha$ treatment in pediatric patients so far. ${ }^{62}$ It is suggested to give BCG vaccine prior starting the immunosuppressive therapy in pediatric patients with RD living in endemic areas. ${ }^{17,63,64}$ Hovewer, BCG vaccination is contraindicated during immunosuppressive treatment, except for contacts of immunosuppressed patients. ${ }^{6}$ The safety of BCG vaccination has not been studied. Kiray et al. ${ }^{65}$ found reduced induration sizes in the tuberculin skin test in 115 patients with JIA on low-dose immunosuppressive drugs. Abe et al. ${ }^{66}$ found similar results in their study, where 20 patients with SLE were evaluated. Freire et al. ${ }^{67}$ have reported that four patients with jSLE on immunsupressive drugs presented miliary tuberculosis. These patients received BCG vaccination at neonatal period and purified protein derive skin tests were negative prior to the treatment.

In the literature, it has been reported that $50 \%$ of 15,805 Kawasaki patients had localized inflammation in BCG vaccination area. ${ }^{68-70}$ For this reason, it is recommended to avoid BCG vaccination in active Kawasaki disease. ${ }^{12}$ On the other hand, two reports have recommended $B C G$ vaccination in young children with $R D$ before the commencement of corticosteroids or biologics. 63,64

Because live tuberculosis bacilli might exist at the site of inoculation at least for 6 months, BCG vaccination is not recommended to pediatric patients with RD receiving corticosteroids, immunosuppressants, or biologics agents. ${ }^{28}$

\section{Poliovirus vaccines}

Patients with inflammatory diseases on immunosuppressants, including systemic corticosteroids should receive the inactive vaccine (Salk), according to the universal immunization schedule, but not the live vaccine (Sabin). ${ }^{71}$ Patients with SLE should not be administered live poliovirus vaccine. ${ }^{72}$

Four of 73 SLE patients showed disease flare following a national vaccination campaign in Israel; however, this report was not confirmed by prospective studies. ${ }^{6}$ In a vaccine campaign to control an epidemic of poliomyelitis in Finland, immunocompromised patients who received the Salk vaccine (inactivated virus) instead of the Sabin vaccine were investigated. The patients had protective titers of neutralizing antibodies against the poliomyelitis virus during the epidemic. ${ }^{73}$ Immunization against poliomyelitis and the post-vaccination level of immunity have been assessed in 136 children with RD who had previously received oral poliovirus vaccine. The proportion of children with RD who were seropositive to all three serotypes was $75.8 \% .^{74,75}$ Although, international guidelines state that live vaccines are contraindicated in patients receiving antiTNF therapy, there is no report of such a patient who received live polio vaccine suffering from any infectious sequelae. ${ }^{76}$

\section{Measles, mumps, rubella (MMR) vaccines}

The MMR vaccine is a live attenuated viral vaccine which is included in national vaccination programs worldwide. The primary vaccine is commonly applied at 12-18 months and the booster is administered among 15 months and 12 years. Usually, primary vaccination is administered before the initiation of the disease. Thus, the essential issue is the safety and immunogenicity of MMR-booster vaccination in pediatric patients with RD. ${ }^{17}$ Borte et al. ${ }^{77}$ 
showed that MMR booster had resulted in good immunogenicity in ten JIA patients, irrespective of MTX and etanercept use. Antibody titres in patients after six months (while on MTX and etanercept treatment) were similar to healthy controls. Several years after the vaccination, measles-specific antibody concentrations were found similar in 18 SLE patients using glucocorticosteroids (mean dose $0.9 \mathrm{mg} / \mathrm{kg}$ / day) and/or other immunosuppressive drugs, compared to healthy controls. ${ }^{51}$ According to Heijstek et al. ${ }^{54}$ protective antibody levels against mumps and rubella up to 10 years after MMR booster vaccination were significantly lower in JIA patients than in healthy controls. Conversely, in other uncontrolled studies involving 15 and 207 JIA patients respectively, MMR booster vaccination did not increase disease activity in patients receiving normal dose MTX, low dose glucocorticosteroids or MTX plus anti-TNF $\alpha$ therapy. ${ }^{77,78}$ The study of Heijstek et al. ${ }^{78}$ showed that the MMR vaccination appears to be safe in JIA. They detected no changes in disease activity, flare occurrence or medication use after the MMR vaccination. No overt measles infections were noted. After adjustments for the possible confounders of JIA type and medication use, risk of active disease was not significantly increased in the vaccinated patients. Two case reports exist about the MMR vaccine safety including a flare of systemic JIA after primary rubella vaccination and idiopathic thrombocytopenic pupura after MMR booster. ${ }^{79,80}$ Although, the recurrence of systemic JIA after rubella vaccination has been reported, no aggrevation of underlying disease or activation of vaccine strains of mumps, measles, and rubella viruses was reported following MMR vaccination in 314 patients with JIA. ${ }^{78,79}$

The efficacy and safety of booster MMR vaccination in JIA patients receiving MTX and/or biologics have recently been further confirmed by a randomized control study ${ }^{81}$.

\section{Varicella vaccines}

The varicella vaccine is produced with live, attenuated virus derived from the OKA strain, and it is administered subcutaneously. ${ }^{6}$ Varicella-zoster virus (VZV) infection is a highly contagious disease in worldwide and an endemic in many countries. ${ }^{82}$ Complicated varicella and herpes zoster infections are very common in pediatric patients with $\mathrm{RD}$ in literature. ${ }^{84,85}$

Serious VZV infections are the major concerns especially in immunocompromised patients receiving corticosteroid, TNF inhibitors, MTX, or calcineurin inhibitors. ${ }^{85-88}$ Therefore, all patients with RD should be screened for sensitivity to the virus before the commencement of such medications. VZV vaccine should also be considered in sensitive patients and, if possible, given at least 3 weeks before the initiation of immunosuppressants or corticosteroids. ${ }^{89}$ Nevertheless, varicella vaccination is contraindicated in patients receiving glucocorticoids at prednisone equivalent doses of $2 \mathrm{mg} / \mathrm{kg} /$ day or $20 \mathrm{mg} /$ day for $>14$ days. ${ }^{90}$

Pileggi et al. ${ }^{83}$ evaluated the immunogenicity and tolerance of the VZV vaccine in one study of 25 sensitive children with RD and 18 healthy controls. All patients were receiving MTX, 13 were also receiving prednisone and five others were using DMARDs. Positive VZV IgG titres were determined in $50 \%$ of the seronegative patients and $72.2 \%$ of the controls in 4-6 weeks after vaccination. No varicella infection and no serious adverse reactions were observed during the follow-up. Evidence on the immunogenicity and safety of the varicella vaccine in patients with SLE is not adequate. ${ }^{91}$ Positive immunity was described in five of six IBD patients using 6 -mercaptopurine and anti-TNF $\alpha$, by Lu et al. ${ }^{92}$; they recommend that live-attenuated vaccine is at least relatively immunogenic, even when immunosuppressants are used.

\section{Yellow fever vaccine}

No studies, related to the immunogenicity of the yellow fever (YF) vaccine in children with RDi were found. The antibody response to YF vaccination has been studied in 91 adult patients with RD and the vaccine showed a good immunogenicity. ${ }^{10}$

\section{Conclusions and Recommendations}

There have been an increasing number of publications and controlled studies in recent years on vaccination in patients with pediatric RD. A small number of vaccination recommandations have been published concerning vaccinations to be applied in these patients. Particularly, the reviews on vaccination in pediatric rheumatologic by EULAR are one 
of the important studies in this regard. In addition, there are other articles published by researchers in Brazil, Japan and Italy. 6,10,17,28,75 As the vaccination experience increases in these group of patients, information on vaccinations will increase and more current standardized vaccination schedule and guidelines will be established. There are important considerations in the vaccination of pediatric rheumatic patients:

1. The vaccination schedule should be questioned at each visit and, be recorded to the vaccination card. Once a rheumatologic disease is diagnosed, the patient's vaccinations should be reviewed immediately and incomplete vaccinations should be completed. If the treatment has already been started, vaccinations should be administered during the period when the disease is at remission and the immunosuppression is at its lowest level.

2. Vaccination should be administered prior to immunosuppression, if possible.

3. Vaccines are effective and safe to prevent the risk of infection when they are administered in accordance with guidelines, and there is no evidence that vaccines lead to rheumatologic diseases or exacerbations in patients with pediatric RD. There is no contraindication for inactive or live vaccines in patients who do not receive immunosuppressive treatment.

4. Inactivated vaccines can be administered to pediatric patients with RD while using glucocorticosteroid, DMARDs and/or antiTNF- $\alpha$ therapy. Inactivated vaccines including inactive influenza virus (IIV), and live virus vaccines should be administered to patients prior to commencement of corticosteroid therapy as indicated on the annual immunization schedule for immunocompetent children, if possible. Inactivated vaccines should be completed $\geq 2$ weeks before and live virus vaccines 34 weeks before the commencement of corticosteroid therapy. ${ }^{11}$

5. To assure the adequate immune response, it is recommended to measure pathogen specific antibody concentrations following vaccination in all pediatric patients with $\mathrm{RD}$ on high-dose glucorticosteroids $(>2$ $\mathrm{mg} / \mathrm{kg} /$ day or $>20 \mathrm{mg} /$ day for 2 weeks) or on rituximab. Measuring pathogen specific antibody concentrations should be considered in patients recieving anti-TNFa treatment.

6. Hydroxychloroquine and sulfasalazine do not affect the immune response and are not considered as immunosuppressive agents. Non-biologic disease modifying agents other than corticosteroids or MTX do not significantly reduce the immune response to inactive vaccines, the response is usually sufficient.

7. It is recommended to adhere to national vaccination guidelines, which have specific focus on geographical conditions, closely interaction with the microflora. Related vaccination schemes would therefore include cholera, diphtheria, Haemophilus influenzae type B (Hib), hepatitis A virus (HAV), $H B V$, Japanese encephalitis, pertussis, pneumococci, poliovirus and meningococci, rabies, tetanus, tickborne encephalitis and typhoid fever, in children with $\mathrm{RD}^{12}$.

8. Immunoglobulin administration may reduce the effectiveness of live vaccines. Therefore, it is necessary to apply live vaccines (i.e. measles, varicella vaccine) at least 3 months following the immunoglobulin administration. If immununglobulin administration is required within 14 days of live vaccine administration, these vaccinations need to be repeated later.

9. Biologic drugs are considered as highly immunosuppressive. Live virus vaccines are contraindicated during and for weeks to months following discontinuation of the biologics. Inactivated vaccines including IIV are recommended during therapy as it is in the annual immunization schedule, and should not be withold because of a concern for an exaggerated inflammatory response. Vaccination status should be documented, and inactivated vaccines (including annual IIV) should be administered 2 weeks before the initiation of biologic drugs, if required. Live virus vaccines should be administered at least 4 weeks before the initiation of biologic therapy, unless contraindicated by a condition or other therapies. Testing for varicella zoster virus and hepatitis B virus should also be considered. ${ }^{93}$ 
10. Seasonal influenza vaccine is safe and immunogenic in adult and pediatric patients with RD. Seasonal influenza vaccine should be recommended annually to these patients. ${ }^{10,15,16,24}$

11. Hepatitis $B$ virus (HBV) vaccine is reported safe and immunogenic in most studies. It is important to check for antibody titers in the follow-up, since they may fall below their protective levels over time in pediatric patients with RD. A booster dose should be considered. ${ }^{10,28,29,30,32,33}$

12. Hepatitis $A$ virus vaccine is safe in patients with JIA. Its effectiveness is similar in both healthy and the ones with RD; however, children with active systemic JIA receiving anti-tumor necrosis factor alpha drugs should be checked for antibody titers. ${ }^{39}$

13. Both PPV and PCV are safe and immunogenic in pediatric patients with $\mathrm{RD}$, although anti-TNF $\alpha$ treatment may reduce antibody concentrations. ${ }^{12,30,44}$

14. Meningococcal vaccine provides sufficient antibody levels, even in patients receiving intense immunosuppressive treatment. Hence, patients with JIA can be vaccinated safely and effectively with the MenC conjugated vaccine. ${ }^{12,45,46}$

15. The tetanus and diphtheria toxoids, and anti-pertussis compound vaccines are safe for children and adults with RD, including immunosuppressive period. Vaccination schedule is the same for healthy children. Nevertheless, antibody titer may fall below the protective level in patients receiving high dose immunosupressives such as glucocorticosteroid or biologic drugs. Antitetanus immunoglobulin may be considered in pediatric patients with RD receiving highdose corticosteroid, immunosuppressants, or biologics. ${ }^{21,47,48,56,57}$

16. Long-term protection against $H P V$ infections in SLE patients is not clear. Larger, controlled studies in jSLE patients are needed to evaluate the immunogenicity of the HPV vaccine in this group. The immunogenicity of the bivalent $H P V$ vaccine in JIA patients are found as adequate up to 12 months after vaccination. Despite lower antibody levels compared to controls, no statistically significant difference in results over time is observed. ${ }^{10,58,61}$

17. In pediatric patients with RD living in endemic areas for tuberculosis, it is suggested to vaccinate prior to initiation of immunosuppressive therapy. However, BCG vaccination is contraindicated during immunosuppressive treatment. Live bacilli are observed at the site of inoculation at least for 6 months, which is a risk factor for disseminated infection in related patients. ${ }^{6,17,28,63,64}$

18. Patients on immunosupressive drugs such as systemic corticosteroids, should receive the inactive vaccine (Salk), according to the universal immunization schedule, but not the live vaccine (Sabin). ${ }^{71}$

19. MMR vaccination appears to be safe in JIA according to recent literature. Protective antibody levels against mumps and rubella up to 10 years following MMR booster vaccination may fall under protective level. ${ }^{17,53,77,78}$ Therefore, the MMR booster vaccine may be needed in pediatric patients with RD over time.

All patients with RD should be screened for sensitivity to the varicella-zoster virus before the commencement of immunosuppressive drugs such as corticosteroids. should be considered in sensitive patients and, if possible, given at least 3 weeks before the inception of such medications. Several studies have found that VZV vaccine is immunogenic in patients using MTX, prednisone and/or biologic agents and no varicella infection is reported in these patients. On the other hand, vaccination with VZV vaccine is contraindicated in patients using high dose immunosuppressives. $83,89,90,92$

\section{REFERENCES}

1. Ada G. Vaccines and vaccination. N Engl J Med 2001; 345: 1042-1053.

2. American Academy of Pediatrics. Active Vaccinations. Pickering LK, Baker JC, Long SS, McMillan JA (eds). Red Book: 2006 Report of the Committee on Infectious Diseases (27th ed). Elk Grove Village IL: American Academy of Pediatrics; 2006: 23-24.

3. Bongartz T, Sutton AJ, Sweeting MJ, Buchan I, Matteson EL, Montori V. Anti-TNF antibody therapy in rheumatoid arthritis and the risk of serious infections and malignancies: Systematic review and meta-analysis of rare harmful effects in randomized controlled trials. JAMA 2006; 295: 2275-2285. 
4. Doran MF, Crowson CS, Pond GR, et al. Frequency of infection in patients with rheumatoid arthritis compared with controls: A population-based study. Arthritis Rheum 2002; 46: 2287-2293.

5. Fessler BJ. Infectious diseases in systemic lupus erythematosus: Risk factors, management and prophylaxis. Best Pract Res Clin Rheumatol 2002; 16: 281-291.

6. Silva CAA, Terreri MTRA, Barbosa CMPL, et al. Immunization consensus for children and adolescents with rheumatic diseases. Bras J Rheumatol 2009; 49: 562-589.

7. Thakur A, Pedersen LE, Jungersen G. Immune markers and correlates of protection for vaccine induced immune responses. Vaccine 2012; 30: 4907-4920.

8. Plotkin SA. Correlates of protection induced by vaccination. Clin Vaccine Immunol 2010; 17: 10551065.

9. Viana PO, Ono E, Miyamoto M, et al. Humoral and cellular immune responses to measles and tetanus: The importance of elapsed time since last exposure and the nature of the antigen. J Clin Immunol 2010; 30: $574-582$.

10. Groot N, Heijstek MW, Wulffraat NM. Vaccinations in paediatric rheumatology: An update on current developments. Curr Rheumatol Rep 2015; 17: 46.

11. American Academy of Pediatrics. Immunization in clinical circumstance. In: Kimberlin DW, Brady MT, Jackson MA, Long SS (eds). Red Book: 2015 Report of the Committee on Infectious Diseases (30th ed). Elk Grove Village, IL: American Academy of Pediatrics; 2015: 74-89.

12. Heijstek MW, Ott de Bruin LM, et al. EULAR recommendations for vaccination in paediatric patients with rheumatic diseases. Ann Rheum Dis 2011; 70: 1704-1712.

13. Maillefert JF, Sibilia J, Toussirot E, et al. Rheumatic disorders developed after hepatitis B vaccination. Rheumatology (Oxford) 1999; 38: 978-983.

14. Rubin LG, Levin MJ, Ljungman P, et al. 2013 IDSA clinical practise guideline for vaccination of the immunocomprimsed host. Clin Infect Dis 2014; 58: 309-318.

15. Kroger AT, Atkinson WL, Marcuse EK, et al. General recommendations on immunization: recommendations of the Advisory Committee on Immunization Practices (ACIP). MMWR Recomm Rep 2006; 55: 1-48.

16. Hak E, Nordin J, Wei F, et al. Influence of high-risk medical conditions on the effectiveness of influenza vaccination among elderly members of 3 large managedcare organizations. Clin Infect Dis 2002; 35: 370-377.

17. Heijstek MW, Ott de Bruin LM, R. Borrow R, et al. Vaccination in paediatric patients with auto-immune rheumatic diseases: A systemic literature review for the European League against Rheumatism evidencebased recommendations. Autoimmun Rev 2011; 11: 112-122.
18. Kanakoudi-Tsakalidou F, Trachana M, Pratsidou-Gertsi P, Tsitsami E, Kyriazopoulou- Dalaina V. Influenza vaccination in children with chronic rheumatic diseases and long-term immunosuppressive therapy. Clin Exp Rheumatol 2001; 19: 589-594.

19. Ogimi C, Tanaka R, Saitoh A, Oh-Ishi T. Immunogenicity of influenza vaccine in children with pediatric rheumatic diseases receiving immunosuppressive agents. Pediatr Infect Dis J 2010; 30: 208-211.

20. Lu Y, Jacobson DL, Ashworth LA, et al. Immune response to influenza vaccine in children with inflammatory bowel disease. Am J Gastroenterol 2009; 104: 444-453.

21. Denman EJ, Denman AM, Greenwood BM, Gall D, Heath RB. Failure of cytotoxic drugs to suppress immune responses of patients with rheumatoid arthritis. Ann Rheum Dis 1970; 29: 220-231.

22. Mamula P, Markowitz JE, Piccoli DA, Klimov A, Cohen $\mathrm{L}$, Baldassano RN. Immune response to influenza vaccine in pediatric patients with inflammatory bowel disease. Clin Gastroenterol Hepatol 2007; 5: 851-856.

23. Malleson PN, Tekano JL, Scheifele DW, Weber JM Influenza immunization in children with chronic arthritis: A prospective study. J Rheumatol 1993; 20: 1769-1773.

24. Shale M, Czub M, Kaplan G. Anti-tumor necrosis factor therapy and influenza: Keeping it in perspective. Therap Adv Gastroenterol 2010; 3: 173-177.

25. Kapetanovic MC, Saxne T, Nilsson JA, Geborek P. Influenza vaccination as model for testing immune modulation induced by anti-TNF and methotrexate therapy in rheumatoid arthritis patients. Rheumatology 2007; 46: 608e11.

26. Vassilopoulos D, Calabrese LH. Management of rheumatic disease with comorbid HBV or HCV infection. Nat Rev Rheumatol 2012; 8: 348-357.

27. Pérez-Alvarez R, Dias-Lagares C, Garcia-Hernández F, et al; BIOGEAS Study Group. Hepatitis B virus (HBV) reactivation in patients receiving tumor necrosis factor (TNF)-targeted therapy: Analysis of 257 cases. Medicine (Baltimore) 2011; 90: 359-371

28. Kobayashi I, Mori M, Yamaguchi K, et al. Pediatric Rheumatology Association of Japan recommendation for vaccination in pediatric rheumatic diseases. Mod Rheumatol 2015; 25: 335-343.

29. Kasapçopur O, Cullu F, Kamburoglu-Goksel A, et al. Hepatitis B vaccination in children with juvenile idiopathic arthritis. Ann Rheum Dis 2004; 63: 11281130.

30. Salinas FG, De Rycke L, Cantaert T, et al. TNF blockade impairs $\mathrm{T}$ cell dependent antibody responses (abstract). Ann Rheum Dis 2009; 68 (Suppl 3): 238.

31. Kuruma KA, Borba EF, Lopes MH, et al. Safety and efficacy of hepatitis B vaccine in systemic lupus erythematosus. Lupus 2007; 16: 350-354.

32. Maritsi D, Vartzelis G, Soldatou A, Garoufi A, Spyridis $\mathrm{N}$. Markedly decreased antibody titers against hepatitis $B$ in previously immunised children presenting with juvenile idiopathic arthritis. Clin Exp Rheumatol 2013; 31: 969-973. 
33. Moses J, Alkhouri N, Shannon A, et al. Hepatitis $\mathrm{B}$ immunity and response to booster vaccination in children with inflammatory bowel disease treated with infliximab. Am J Gastroenterol 2012; 107: 133-138.

34. Aytac MB, Kasapcopur O, Aslan M, Erener-Ercan T, Cullu-Cokugras F, Arisoy N. Hepatitis B vaccination in juvenile systemic lupus erythematosus. Clin Exp Rheumatol 2011; 29: 882-886.

35. Beran J, Dedek P, Stepanova V, Spliio M, Pozler O. Safety and immunogenicity of a combined vaccine against hepatitis A and B in patients with autoimmune hepatitis. Cent Eur J Public Health 2005; 13: 20-23.

36. Geier MR, Geier DA. A case-series of adverse events, positive re-challenge of symptoms, and events in identical twins following hepatitis $B$ vaccination: Analysis of the Vaccine Adverse Event Reporting System (VAERS) database and literature review. Clin Exp Rheumatol 2004; 22: 749-755.

37. Moxey-Mims MM, Preston K, Fivush B, McCurdy F. Heptavax-B in pediatric dialysis patients: Effect of systemic lupus erythematosus. Chesapeake Pediatric Nephrology Study Group. Pediatr Nephrol 1990; 4: 171-173.

38. Russo RA, Rosenzweig SD, Katsicas MM. Hepatitis A-associated macrophage activation syndrome in children with systemic juvenile idiopathic arthritis: Report of 2 cases. J Rheumatol 2008; 35: 166e8.

39. Erguven M, Kaya B, Hamzah OY, Tufan F. Evaluation of immune response to hepatitis $\mathrm{A}$ vaccination and vaccine safety in juvenile idiopathic arthritis. $\mathrm{J}$ Chin Med Assoc 2011; 74: 205-208.

40. Centers for Disease Control and Prevention. Recommended Immunization Schedule for Children and Adolescents Aged 18 Years or Younger, United States, 2017. Available at: https://www.cdc.gov/ vaccines/schedules/hcp/imz/child-adolescent.html (Accessed June 21, 2017)

41. Nuorti JP, Whitney CG; Centers for Disease Control and Prevention (CDC). Prevention of pneumococcal disease among infants and children use of 13-valent pneumococcal conjugate vaccine and 23 -valent pneumococcal polysaccharide vaccine-recommendations of the Advisory Committee on Immunization Practices (ACIP). MMWR Recomm Rep 2010; 59: 1-18.

42. Advisory Committee on Immunization Practices. Preventing pneumococcal disease among infants and young children. Recommendations of the Advisory Committee on Immunization Practices (ACIP). MMWR Recomm Rep Oct 2000; 49: 1-35.

43. Centers for Disease Control and Prevention (CDC). Use of 13-valent pneumococcal conjugate vaccine and 23-valent pneumococcal polysaccharide vaccine for adults with immunocompromising conditions: recommendations of the Advisory Committee on Immunization Practices (ACIP). MMWR Morb Mortal Wkly Rep 2012; 61: 816-819.

44. Farmaki E, Kanakoudi-Tsakalidou F, Spoulou V, et al. The effect of anti-TNF treatment on the immunogenicity and safety of the 7-valent conjugate pneumococcal vaccine in children with juvenile idiopathic arthritis. Vaccine 2010; 28: 5109-5113.
45. Zonneveld-Huijssoon E, Ronaghy A, van Rossum MA, et al. Safety and efficacy of meningococcal $\mathrm{C}$ vaccination in juvenile idiopathic arthritis. Arthritis Rheum 2007: 56; 639-646.

46. Stoof SP, Heijstek MW, van der Klis F, et al. Kinetics of the long-term antibody responseafter meningococcal $C$ vaccination in patients with juvenile idiopathic arthritis: A retrospective cohort study. Ann Rheum Dis 2014; 73: 728-734.

47. Snowden N. Immunization of immunosuppressed patients with rheumatic diseases. Top Rev . 2007; 12 $1-12$.

48. American Academy of Pediatrics. Immunization in special clinical circumstances. In: Pickering LK (ed). 2003 Red Book. Report of the Committee on Infectious Diseases (26th ed). Elk Grove Vilage: American Academy of Pediatrics; 2003: 54-81.

49. Centers for Disease Control and Prevention (CDC). Pertussis. United States, 1997-2000. MMWR Morb Mortal Wkly Rep 2002; 51: 73-76.

50. de Carvalho AP, Pereira EM. Acellular pertussis vaccine for adolescents. J Pediatr (Rio J) 2006; 82: S15-S24.

51. Miyamoto M, Ono E, Barbosa C, et al. Vaccine antibodies and $\mathrm{T}$ - and $\mathrm{B}$-cell interaction in juvenile systemic lupus erythematosus. Lupus 2011; 20: 736744.

52. Hoyeraal HM, Mellbye OJ. Humoral immunity in juvenille rheumatoid arthritis. Ann Rheum Dis 1974; 33: $248-254$

53. Kashef S, Ghazizadeh F, Derakhshan A, Farjadian $S$, Alyasin S. Antigen-specific antibody response in juvenile-onset SLE patients following routine immunization with tetanus toxoid. Iran J Immunol 2008; 5: 181-184.

54. Heijstek MW, van Gageldonk PG, Berbers GA, Wulffraat NM. Differences in persistence of measles, mumps, rubella, diphtheria and tetanus antibodies between children with rheumatic disease and healthy controls: a retrospective cross-sectional study. Ann Rheum Dis 2012; 71: 948-954.

55. Bingham CO III, Looney RJ, Deodhar A, et al Immunization responses in rheumatoid arthritis patients treated with rituximab: Results from a controlled clinical trial. Arthritis Rheum 2010; 62: 64-74.

56. Tay L, Leon F, Vratsanos G, Raymond R, Corbo M. Vaccination response to tetanus toxoid and 23-valent pneumococcal vaccines following administration of a single dose of abatacept: A randomized, open-label, parallel group study in healthy subjects. Arthritis Res Ther 2007; 9: R38.

57. Koshcheeva IuV, Kharit SM, Kalinina NM. Specific features of diphtheria vaccinal process in children with rheumatic diseases. Zh Mikrobiol Epidemiol Immunobiol 2001; 6: 44-49.

58. Heijstek MW, Scherpenisse M, Groot N, et al. Immunogenicity and safety of the bivalent HPV vaccine in female patients with juvenile idiopathic arthritis: A prospective controlled observational cohort study. Ann Rheum Dis 2014; 73: 1500-1507. 
59. Klumb EM, Araujo Jr ML, Jesus GR, et al. Is higher prevalence of cervical intraepithelial neoplasia in women with lupus due to immunosuppression? J Clin Rheumatol 2010; 16: 153-157.

60. Soybilgic A, Onel KB, Utset T, Alexander K, Wagner-Weiner L. Safety and immunogenicity of the quadrivalent HPV vaccine in female systemic lupus erythematosus patients aged 12 to 26 years. Pediatr Rheumatol Online J 2013; 11: 29.

61. Mok CC, Ho LY, Fong LS, To CH. Immunogenicity and safety of a quadrivalent human papillomavirus vaccine in patients with sys- temic lupus erythematosus: A case-control study. Ann Rheum Dis 2013; 72: 659-664.

62. Armbrust W, Kamphuis SS, Wolfs TW, et al. Tuberculosis in a nine-year-old girl treated with infliximab for systemic juvenile idiopathic arthritis. Rheumatology (Oxford) 2004; 43: 527-529.

63. Lovell DJ, Reiff A, Ilowite NT, et al. Safety and efficacy of up to eight years of continuous etanercept therapy in patients with juvenile rheumatoid arthritis. Arthritis Rheum 2008; 58: 1496-1504.

64. Ruperto N, Lovell DJ, Quartier P, et al. Long-term safety and efficacy of abatacept in children with juvenile idiopathic arthritis. Arthritis Rheum 2010; 62: 1792-1802.

65. Kiray E, Kasapcopur O, Bas V, et al. Purified protein derivative response in juvenile idiopathic arthritis. J Rheumatol 2009; 36: 2029-2032.

66. Abe T, Homma M. Immunological reactivity in patients with systemic lupus erythematosus. Humoral antibody and cellular immune responses. Acta Rheumatol Scand 1971; 17: 35-46.

67. Freire PS, Montoni JD, Ribeiro AS, Marques HH, Mauad T, Silva CA. Miliary tuberculosis: A severe opportunistic infection in juvenile systemic lupus erythematosus patients. Rev Bras Reumatol 2016; 56: 274-279.

68. Weinstein M. Inflammation at a previous inoculation site: an unusual presentation of Kawasaki disease. CMAG J 2006; 174: 459-460.

69. Antony D, Jessy PL. Involvement of BCG scar in Kawasaki disease. Indian Pediatr 2005; 42: 83-84.

70. Uehara R, Igarashi H, Yashiro M, Nakamura Y, Yanagawa $\mathrm{H}$. Kawasaki disease patients with redness or crust formation at the Bacille Calmette-Guerin inoculation site. Pediatr Infect Dis J 2010; 29: 430-433.

71. Davies K, Woo P; British Paediatric Rheumatology Group. Immunization in rheumatic diseases of childhood: an audit of the clinical practice of British Paediatric Rheumatology Group members and a review of the evidence. Rheumatology (Oxford) 2002; 41: 937-941.

72. O' Neill SG, Isenberg DA. Immunizing patients with systemic lupus erythematosus: A review of effectiveness and safety. Lupus 2006; 15:778-783.

73. Stenvik M, Hovi L, Siimes MA, Roivainen M, Hovi T. Antipolio prophylaxis of immunocompromised children during a nationwide oral poliovirus campaign. Pediatr Infect Dis J 1987; 6: 1106-1110.
74. Tarasova AA, Kniagina ON, Kalashnikova NA, Kostinov MP, Erukhimov VL. Immunity to poliovirus after vaccination of children with type 1 diabetes mellitus and rheumatic diseases. Zh Mikrobiol Epidemiol Immunobiol 2009; 5: 52-56.

75. Dell'Era L, Esposito S, Corona F, Principi N. Vaccination of children and adolescents with rheumatic diseases. Rheumatology 2011; 50: 1358-1365.

76. Badsha $\mathrm{H}$, Daher M, Edwards CJ. Live polio vaccine exposure while receiving anti-TNF therapy for reactive arthritis. Int J Rheum Dis 2010; 13: 184-186.

77. Borte S, Liebert UG, Borte M, Sack U. Efficacy of measles, mumps and rubella revaccination in children with juvenile idiopathic arthritis treated with methotrexate and etanercept. Rheumatology (Oxford) 2009; 48: 144-148.

78. Heijstek MW, Pileggi GC, Zonneveld-Huijssoon E, et al. Safety of measles, mumps and rubella vaccination in juvenile idiopathic arthritis. Ann Rheum Dis 2007; 66: $1384-1387$

79. Korematsu S, Miyahara H, Kawano T, et al. A relapse of systemic type juvenile idiopathic arthritis after a rubella vaccination in a patient during a long-term remission period. Vaccine 2009; 27: 5041-5042.

80. Drachtman RA, Murphy S, Ettinger LJ. Exacerbation of chronic idiopathic thrombocytopenic purpura following measles-mumps-rubella immunization. Arch Pediatr Adolesc Med 1994; 148: 326-327.

81. Heijstek MW, Kamphuis S, Armbrust W, et al. Effects of the live attenuated measles-mumps- rubella booster vaccination on disease activity in patients with juvenile idiopathic arthritis in a randomized trial. JAMA 2013; 309: 2449-2456.

82. Heininger U, Seward JF. Varicella. Lancet 2006; 368 : 1365-1376.

83. Pileggi GS, de Souza CB, Ferriani VP. Safety and immunogenicity of varicella vaccine in patients with juvenile rheumatic diseases using methotrexate and corticosteroids. Arthritis Care Res 2010; 62: 903-906.

84. Angit C, Daly BM. Disseminated varicella zoster infection in a patient with rheumatoid arthritis treated with methotrexate. Clin Exp Dermatol 2009; 34: 453454.

85. Strangefeld A, Listing J, Herzer P et al. Risk of herpes zoster in patients with rheumatoid arthritis treated with anti-TNF-alpha agents. J Am Med Assoc 2009; 301: 737-744.

86. Bijl M, Agmon-Levin N, Dayer JM, Israeli E, Gatto M, Shoenfeld Y. Vaccination of patients with auto-immune inflammatory rheumatic diseases requires careful benefit-risk assessment. Autoimmune Rev 2012; 11: 572-576.

87. Kinder AJ, Hassell AB, Brand J, Brownfield A, Grove M, Shadforth MF. The treatment of inflammatory arthritis with methotrexate in clinical practice: treatment duration and incidence of adverse drug reactions. Rheumatology (Oxford) 2005; 44: 61-66.

88. Vonkeman H, ten Napel C, Rasker H, van de Laar M. Disseminated primary varicella infection during infliximab treatment. J Rheumatol 2004; 31: 25172518. 
89. Marin M, Guris D, Chaves SS, Schmid S, Seward JF; Advisory Committee on Immunization Practices, Centers for Disease Control and Prevention (CDC). Prevention of varicella: recommendations of the Advisory Committee on Immunization Practices (ACIP). MMWR Recomm Rep 2007; 56: 1-40.

90. Gershon AA, Steinberg SP, Gelb L. Live attenuated varicella vaccine use in immunocompromised children and adults. Pediatrics 1986; 78(4 Pt 2): 757-762.
91. O’ Neill SG, Isenberg DA. Immunizing patients with systemic lupus erythematosus: A review of effectiveness and safety. Lupus 2006; 15: 778-783.

92. Lu Y, Bousvaros A. Varicella vaccination in children with inflammatory bowel disease receiving immunosuppressive therapy. J Pediatr Gastroenterol Nutr 2010; 50:562-565.

93. Le Saux N; Canadian Paediatric Society, Infectious Diseases and Immunization Committee. Biologic response modifiers to decrease inflammation: Focus on infection risks. Paediatr Child Health 2012; 17: 147-154. 